\title{
Learning Computer Science Fundamentals through Virtual Environments
}

\author{
James Braman ${ }^{1}$, Giovanni Vincenti ${ }^{2}$, Ana Maria Arboleda Diaz ${ }^{3}$, \\ and Andrew Jinman ${ }^{4}$ \\ ${ }^{1}$ Department of Computer and Information Sciences \\ Towson University, \\ Towson, MD U.S.A. \\ Jbraman@towson. edu \\ ${ }^{2}$ Research and Development \\ Gruppo Vincenti, S.r.l. \\ Rome, Italy \\ Giovanni.vincenti@gmail.com \\ ${ }^{3}$ Department of Education \\ Towson University, \\ Towson, MD U.S.A. \\ Amarboleda81@gmail.com \\ ${ }^{4}$ Immersive Learning Producer \\ Twofour Learning \\ Plymouth, U.K. \\ Andrew.Jinman@twofour.co.uk
}

\begin{abstract}
Utilizing the inherent visual qualities of immersive virtual environments can be advantageous for educating students on particular topics. Basic fundamentals in Computer Science curriculums often can be difficult for students to grasp due to high levels of abstraction and various theoretical frameworks used to describe important structures. Virtual worlds such as Second Life ${ }^{\circledR}$ provide a unique medium for education, allowing for the visualization of concepts coupled with the interaction potential that this environment allows. Briefly discussing traditional methods for teaching fundamental topics in several courses, we discuss how Second Life can be integrated into classroom instruction for the benefit of student understanding of complex materials. We outline some of our preliminary observations and student feedback as we begin to use virtual environments in new innovative ways.
\end{abstract}

Keywords: Virtual worlds, Immersive education, Second Life.

\section{Introduction}

Educating students in various complex topics can be a challenge, often invoking educators to develop creative and innovative strategies to convey key concepts. Changes from traditional pedagogy has often been guided by the changing hand 
technology has brought to the education forefront. These changes have brought new methods and structures of communication, learning, interaction and collaboration. Newer strategies of teaching through virtual worlds can be used as a tool to enhance learning. Many virtual and artificial spaces create environments that bypass the normal physical boundaries associated with real life and real time face-to-face interaction [1]. Although users typically are comfortable with these new virtual forms of interacting, more often educators are not experienced in utilizing these virtual spaces. In this paper we focus on several courses where we have used Second Life® on a preliminary basis in order to gather student feedback on using this medium for future activities. We also discus some of our current work in developing virtual interactive objects for teaching. Certain topics in computer science are essential for students to grasp if they are to have a solid foundation in this discipline. In the United States student enrolment for computer science and computer information systems majors have been on the decline, which should illustrate a need to make educational changes in this area [2]. It is our desire to see virtual environments like Second Life utilized more often to engage and encourage students entering this field and to help students learn fundamental topics.

\section{Teaching in Second Life}

The question is often raised as to what makes teaching within Second Life superior than a standard classroom or using standardized e-learning methods for distance education. Second life is a multi-user visual three-dimensional medium where concepts need not be solely described by text, verbal communication or through static images. Even though by using other modes of education, students and teachers can engage in dynamic communication, they are often limited by resources when discussing the topic at hand. This is certainly true when the need to visualize a topic would be beneficial or when a "hands-on" approach would be useful compared to only a discussion. In Second life, teachers can immerse students within a topic (i.e. walking students through a giant computer for example, instead of merely talking about it or viewing pictures in a book or on the Web). Both students and teachers are represented in these spaces through an avatar, where they can interact within the environment and with other users. Unlike learning in a text based setting, students can still interact in a similar fashion as a real classroom since they can still see and interact with the instructor. Interactions and relations between students and teachers are often enhanced by certain verbal and nonverbal visual cues observed by students in the traditional classroom [3]. Unlike many online course deliveries that depend on text-based, audio or pre-recorded video content, Second life maintains the necessary "face-to-face" interaction as in the real world, even if these interactions are being simulated. One can still "see" and interact in a more "natural" manner then what is currently permitted in current E-learning technologies. Online virtual courses could perhaps be beneficial for students unable to perform in a regular classroom or those that have dropped out for any number of personal reasons [4]. Teaching within Second Life presents a completely new set of obstacles to overcome in comparison to traditional teaching practices. However it is largely reported that many are experimenting within Second Life, with more than 150 academic institutions within 
the environment [5]. We can expect to see many innovative approaches to teaching in Second Life in the near future.

Second Life possesses several characteristics considered beneficial for teaching; Cooper et al, noted the use of graphic animation to possess significant benefits for teaching and learning. "We believe that using 3D animation programming in a preCS1 course provides real benefits in teaching fundamental programming and problem solving concepts. A major benefit is the high level of student interest and involvement" [6]. The OU (Open University) Schome Park project was initiated to evaluate the educational potential and pitfalls of Teen Second Life, using 149 National Association of Gifted and Talented (NAGTY) students. In a recent study the project reported "That the level of engagement was comparable to other media. Of the $68 \%$ of students visiting the island, $41 \%$ spent more than an hour." [7]. However using Second Life as a teaching environment also introduces many new variables and issues, such as barriers to entry (usability issues and communication methods), student expectations and content complexity as observed in similar studies. "Student expectations for the software were high due to the production values seen in commercial video games" [8].

Once they understand the environment, students begin to adapt to it, overcoming these difficulties. Rebecca Nesson (instructor for CyberOne: Law in court of public opinion at Harvard Law School) found students adopted new classroom norms, diverting form traditional formalities of the classroom (for example raising your hand and waiting for one person to finish talking before you begin) [9]. Understanding the environment and its capabilities enables educators to prepare for and understand the level of change required, in respect to traditional teaching practices. Portions of courses may well benefit from the use of Second Life as a delivery mechanism, but "the use of 3D environments also introduces unnecessary confusion and complexity to the content material covered" [8]. For this reason it is important to instruct students on how to use Second Life as a tool and make sure they understand how to use the program successfully. It is the Author's observation that Second Life can be used in combination with face-to-face meeting to help visualize a topic and enhance learning.

\section{Course Discussions}

The courses discussed for this article cover a wide range of topics, as well as serving an eclectic student population. The first course, "Computers and Creativity", is designed for non-computer science majors, and is part of a general set of educational requirements. The second course, "Information Technology for Business" is also part of the core general requirements, but it attracts mainly students from business and economics tracks. The presence of students majoring in computing-related disciplines taking these two courses is not significantly different from their concentration in other core general education courses.

The other courses that we will discuss are part of degree requirements for the department of Computer and Information Sciences. This department has two tracks, "Computer Information Sciences" (CIS) and "Computer Science" (CS). The first degree focuses primarily on business and management aspects. Although programming is required, it usually does not play a major role in the courses. The second degree does rely heavily on programming, as the students are more involved 
with the technical aspects of computing. The third course on which we will focus is called "Data Organization", which is part of the CIS track. The fourth course is "Programming Languages: Design and Implementation", and it is part of the CS track. Both these courses require as a prerequisite the satisfactory completion of two programming courses.

\subsection{Computers and Creativity}

Second Life is being utilized in an introductory computer science course titled "Computers and Creativity" [10]. The main focus of this course is to give students basic instruction in relevant computer skills while emphasizing the creative nature of computers and technology. This traditional class is designed with a typical lecture and lab component where students learn the basics of several multimedia programs, web design and animation. The course content and creative goals match well with the expressive power and creative nature of Second Life by enabling students to extend conceptualized versions of themselves and their work into these creative virtual spaces. A preliminary investigation into taking this course's content into Second Life yielded interesting student insight into the advantages of using Second Life for particular classroom activities and to enhance class discussions [11]. Some activities for this class included in-world student projects, virtual field trips, basic building and scripting. Second Life is also the basis for many class discussions. Second Life based Machinima (filming in 3D worlds) has also been incorporated into class topics as it can be used to illustrate this type of filming technique and video editing [12].

\subsection{Information Technology for Business}

This course aims to provide a foundation of computer science concepts for students who will pursue a career in a business related field, but it is open to other students and is part of the general education requirements set for graduation. This course covers a wide range of information, spanning from concepts of computing applied to industry to the more technical details of the inner workings of hardware components. Class activities also include a team project that focuses on research of current topics in Information Technology. Generally, the students are given an array of topics from which they can choose. The topics are chosen in order to offer a range of interests as varied as the backgrounds of the students. Topics that reoccur every semester include technological advancements in business environments, such as the New York Stock Exchange, robotics and space exploration, and finally social matters such as the influence of computers on psychological and sociological aspects of one's life.

Second Life as a team research project is very fitting for this course as it can cross many of these topic areas often discussed. The main obstacle to this project is the little knowledge that students have about Second Life. It is our experience that the average student taking this course has never heard of Second Life, and when introduced to it, they are very reluctant to pursue further involvement in it without further information. In order to introduce Second Life into the course we use two avenues. First, the instructor shows an overview of virtual worlds as an alternative to the World Wide Web, including a more direct visualization of the content as well as social behaviors. The second happens through the research of peers. The topic focusing on psychological and social aspects of computing is picked every semester, given the high concentration of psychology students usually enrolled in the course. 
The potential of Second Life for a course such as this could be substantial. The visualization alone of concepts of computing, as well as potential simulations that may illustrate ideas such as hard disk management or robotics in workplaces may lead to many educational benefits. In situations where equipment cannot be used due to expense or resource allocation, simulations via second Life could be a useful alternative. The main concern that instructors should have is to let the students experience this world through a constructive approach.

\subsection{Data Organization}

A course that is extremely important to CIS majors is "Data Organization". This course emphasizes to students the idea of a programming language as a means to solve logical problems by introducing abstract constructs whose properties and behaviors resolve programmatic challenges. The students coming into this course have generally completed their pre-requirement of two programming courses. The content of the course is split into two major areas: data organization in primary memory, and data organization in secondary storage. Since it would be impossible to review every type of file format present in the world of computing, the students are exposed to an introduction to XML technologies as a class, and then they are required to work individually on other file formats. The formats that they can use as research topic are agreed upon in conjunction with the instructor. The material found for each data structure is then presented to the class at the end of the semester.

The most important content area of this course revolves around data structures residing in primary memory. Our approach to data structures presents a dual methodology. Students are generally first exposed to in-class lectures and then they are directed to online tutorials and visual representations. The evaluation of student performance for this portion of the course is based on tests, quizzes and one teamprogramming project. The students are encouraged to interact among themselves using the online interface of the course, a relatively simple content management system. Although it contains features such as chat and message boards, the students do not make use of these resources. This can be attributed to the high frequency with which they interact directly face-to-face.

Currently, the course includes a group project that simulates the scheduling of package deliveries for an international shipping company. The program accepts packages given to the driver from the main warehouse and then places them in order for the driver to deliver. The order is based on priority and date of shipping. A MinHeap powers the program. This structure organizes objects by allowing the ones with the smallest value to be towards the front, and the ones with the higher ones towards the back. The only element with which the user can interact is the front-most one, thus the one with the lowest value associated with it. The data structure that serves the opposite task, the higher the number, the higher the priority is called a MaxHeap. In this case, the lower the number associated with a package, the higher the priority. Given two packages with the same priority, the driver will deliver the one that was shipped earlier. This project does not take into consideration the calculation and optimization of routes. The students are expected to create this program using $\mathrm{C}++$. 
The project can easily be ported to Second Life by adding a visualization element that will animate the packages and re-order them according to the requirements outlined above. As this course is part of the CIS curriculum, most students who are enrolled report a strong dislike of programming. Although the CIS track is not oriented towards programming, the students are still required to do so for this course. The problem arises when a group of students working together contains one member who is proficient at coding and often ends up as the sole worker for the duration of the project. The majority of the students who implicitly choose not to participate to the coding part of the project generally have little or no idea of how the data structures required for this program really work. It is our belief that the graphic nature of Second Life will help such students become more involved with the coding, as they will be able to visually analyze their work. Moreover, the students who will still choose not to collaborate will be able to at least visualize the outcome of the code. In this case Second Life will serve as an aide to the visualization and animation of algorithms.

\subsection{Programming Languages}

A general programming language course is offered to upper level (i.e. the junior or senior level), computer science students which aims toward a broad study of different types of programming languages and methods. At this point in the student's academic career, most are quite proficient in either the Java or $\mathrm{C}++$ language. This course forces students to look at other languages that are older and/or languages that are not often used or taught in the mainstream that may be considered more obscure. Usually learning these languages can be both difficult and time consuming, requiring many hours of hands on activities in order to grasp important programming concepts. Trying to demonstrate much of this material in the short span of the semester can be a challenge.

Second Life in this instance could help speed up the process of at least demonstrating one extra language to students. Using the Linden Scripting Language (LSL) students can practice programming while being able to observe immediately what effect their code will have within the Second Life world. Although LSL is not a required language for a general undergraduate programming langue course, some students have opted to research on this language for semester projects to investigate this new way of learning to program in a virtual environment. Students can also use LSL to create scripts and insert them into various created objects in the world to observe their behavior. The Linden Scripting Language possesses many qualities and features required to create elaborate and useful scripts where students can be encouraged to test their skills. This environment can encourage both a collaborative and competitive space where students can ask other residents for help on LSL (if needed) and compete with other students in creating scripted objects. One other aspect of this particular class is the group project presentations, which when completed are uploaded on the web for other students to review and take notes. A long term goal for this class is to have students also upload their programming projects to Second Life along with a virtual poster presentation of the project so others can observe and learn. 


\section{Current Work}

After gaining insight on student perception of Second Life and from our observation of how it was used in these courses, we are beginning to build interactive objects to enhance learning. We are also starting to refine our teaching strategies based on our observations. Besides the interactive social aspects of this platform and its potential for creating an online persistent virtual classroom, we want students to use Second Life to better understand key fundamental data structures and concepts. In the lower division courses, we can take students on a series of virtual fieldtrips to interact with large scale 3D components; or easily visit places in-world that have a presence in Second Life like Dell or IBM that would be of interest to business students. The material is relatively easy to present through such medium, and many visualizations already exist in this world to enhance the material of certain courses. For example, the architecture of the computer could be easily explained by adapting the lecture notes to the Dell Island, which includes a detailed model of a computer's hardware.

Other potential usage resides in 3D interactive data structures, where students can view, build and manipulate these concepts "hands-on". Research suggests [13] that, as students visualize and animate algorithms in different ways, online tutorials for this subject are not as effective as for other subjects. These objects in Second Life would not be merely animations, but interactive and "real" to the Second Life world. Figure 1 illustrates the beginnings of a large scale interactive stack that contains information about its operation. Both memory and data is represented visually in a way where the avatar can interact. Figure 2 shows an interactive working calculator based on post fixed notation. As an avatar enter numbers on the calculator, colored blocks appear, containing the number and is placed on a Stack that they can see and manipulate. Once they choose an operation to perform on the numbers, they are taken out of memory (visually), calculated (visually and displayed via text) and results are placed back into the stack. This object can help student "see" and interact with a visual stack algorithm. Figure 3 illustrates a poster in Second Life that provides students with basic information on a standard Queue and Figure 4 is a large scale interactive Tree structure.

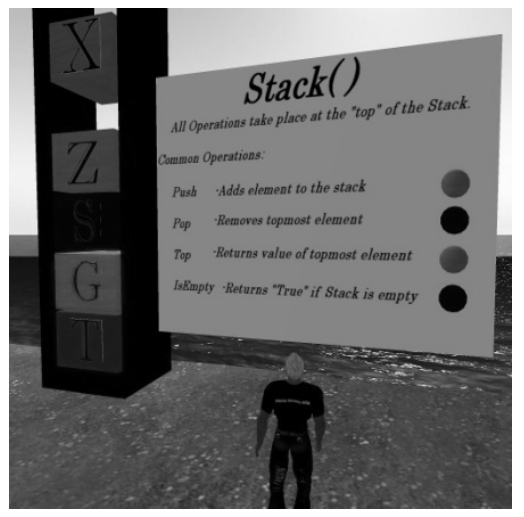

Fig. 1.

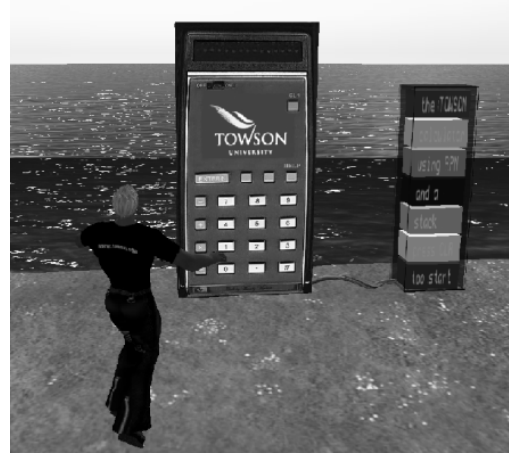

Fig. 2. 


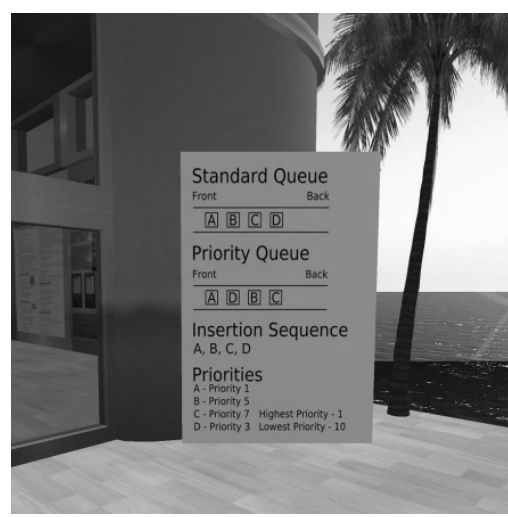

Fig. 3.

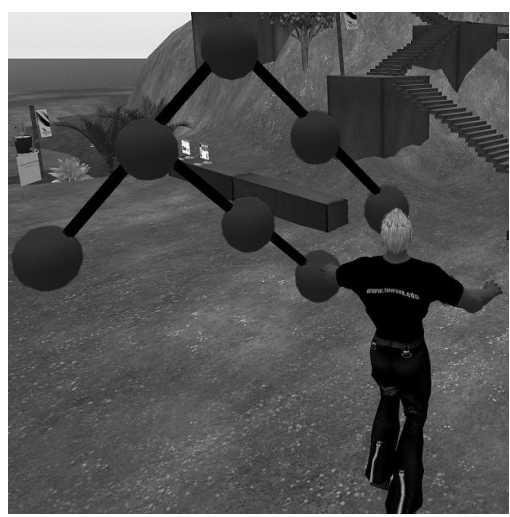

Fig. 4.

This virtual environment though has the potential of becoming a great asset as a method of development and evaluation of student work. Students could individually or collaboratively work on visually based assignments and instructors could evaluate their understanding of a concept based on their build or by their script programmed for a project.

\section{Future Work}

As we experiment with Second life further for educational purposes, our aim is to develop more interactive components. Not only can we simulate computer components and virtual lab spaces for some introductory courses, we can create many more data structures and abstractions for computer science students. We hope to conduct further studies in the classroom and evaluate the effectiveness of Second life as a teaching tool for this type of content.

\section{Conclusions}

Utilizing our experiences with these courses and student feedback we have outlined how virtual worlds like Second Life can be used to enhance student learning and encourage students in computer science. Fundamental concepts in this area of study can be taught not only in the traditional classroom or online, but also in more dynamic settings through platforms like virtual worlds. It is our hope that this paper will encourage other educators to realize the potential of Second Life in more complex domains such as computer science for simulation and interactive learning.

The methods of content delivery are changing along with changes in technology. It is essential that we adapt our sense of the "classroom" to encompass a broader scope of meaning that is not dependant on physical settings or metaphors. As students use multimedia and other visually enhanced computer technology for learning, students may need to shift from learning only through text books and discussion, but through immersion. We believe that these environments will truly enhance teaching and 
learning in the future. The possibilities and the fundamental mechanisms of the environment allow creativity to flourish while enhancing education. Computer science fundamentals are only one important area that can be taught through a virtual world. These spaces are not just pixels on a screen, but represent a new classroom where students can learn in new and innovative ways.

Acknowledgments. This project has been supported by a Teaching Innovation Grant from the Faculty Development and Research Committee of Towson University. We would also like to thank Mandy Medusa for her programming assistance in this project.

\section{References}

1. Trajkovski, G., Collins, S., Braman, J., Goldberg, M.: Coupling Human and Non-Human Agents. In: The AAAI Fall Symposium: Interaction and Emergent Phenomena In Societies of Agents, Arlington, VA (October 2006)

2. Lomerson, W., Pollacia, L.: Declining CIS Enrolment: An Examination of Pre-College Factors. In: Proceedings of the 22nd Annual Information Systems Education Conference (2005)

3. Richmond, V., McCroskey, J.: Nonverbal Behavior in Interpersonal Relations, 5th edn. Pearson Education, London (2004)

4. Livingston, P.: E-learning Gets Real. Technology \& Learning 28(4), 20-22 (2008)

5. Simteach Wiki (2008), http: / / www . simteach. com/wiki (retrieved July 15, 2008)

6. Cooper, S., Dann, W., Pausch, R.: Using Animated 3D Graphics to Prepare Novices for CS1. Computer Science Education 13(1) (2003)

7. Freitas, S.: Emerging trends in serious games and virtual worlds. Emerging technologies for learning 3, 58-72 (2008)

8. Elliott, J., Adams, L., Bruckman, A.: No Magic Bullet: 3D Video Games in Education. In: Proceedings of ICLS 2002, Seattle, Washington (2002)

9. Nesson, R.: (Harvard Law School) Interview, http://ilamont.blogspot.com/2007/05/ interview-harvards-rebecca-nesson.html

10. Braman, J., Jinman, A., Trajkovski, G.: Towards a Virtual Classroom: Investigating Education in Synthetic Worlds. In: The AAAI Fall Symposium. Emergent Agents and Social and Organizational Aspects of Intelligence, Arlington, VA (November 2007)

11. Braman, J., Jinman, A., Trajkovski, G.: Exploring Virtual Worlds as an Extension to Classroom Learning. In: International Conference on Information Society, Merrillville, Indiana (October 2007)

12. Filimon, S.M.: The Handbook of Research on Computational Arts and Creative Informatics. In: Braman, J., Vincenti, G. (eds.) Information Science Reference (2009)

13. Douglas, S., McKeown, D., Hundhause, C.: Exploring Human Visualization of Computer Algorithms. In: Proceedings of Graphics Interface, Toronto, May 1996, vol. 196, pp. 9-16 (1996) 